\title{
Viscosity approximation methods for nonexpansive mappings in CAT(0) spaces
}

\author{
Rabian Wangkeeree ${ }^{*}$ and Pakkapon Preechasilp
}

${ }^{*}$ Correspondence: rabianw@nu.ac.th

Department of Mathematics, Faculty of Science, Naresuan University, Phitsanulok, 65000, Thailand

\section{Springer}

\section{Abstract}

The purpose of this paper is to study the strong convergence theorems of Moudafi's viscosity approximation methods for a nonexpansive mapping $T$ in CAT(0) spaces without the property $\mathcal{P}$. For a contraction $f$ on $C$ and $t \in(0,1)$, let $x_{t} \in C$ be the unique fixed point of the contraction $x \mapsto t f(x) \oplus(1-t) T x$; i.e.,

$$
x_{t}=t f\left(x_{t}\right) \oplus(1-t) T x_{t}
$$

and

$$
x_{n+1}=\alpha_{n} f\left(x_{n}\right) \oplus\left(1-\alpha_{n}\right) T x_{n}, \quad n \geq 0,
$$

where $x_{0} \in C$ is arbitrarily chosen and $\left\{\alpha_{n}\right\} \subset(0,1)$ satisfies certain conditions. We prove that the iterative schemes $\left\{x_{t}\right\}$ and $\left\{x_{n}\right\}$ converge strongly to the same point $\tilde{x}$ such that $\tilde{x}=P_{F(T)} f(\tilde{x})$, which is the unique solution of the variational inequality (VIP)

$$
\langle\overrightarrow{\tilde{x} f \tilde{x}}, \overrightarrow{x \tilde{x}}\rangle \geq 0, \quad x \in F(T)
$$

By using the concept of quasilinearization, we remark that the proof is different from that of Shi and Chen in J. Appl. Math. 2012:421050, 2012. In fact, strong convergence theorems for two given iterative schemes are established in CAT(0) spaces without the property $\mathcal{P}$.

Keywords: viscosity approximation method; nonexpansive mapping; variational inequality; CAT(0) space; common fixed point

\section{Introduction}

Let $(X, d)$ be a metric space. A geodesic path joining $x \in X$ to $y \in X$ (or, more briefly, a geodesic from $x$ to $y$ ) is a map $c$ from a closed interval $[0, l] \subset \mathbb{R}$ to $X$ such that $c(0)=x$, $c(l)=y$, and $d\left(c(t), c\left(t^{\prime}\right)\right)=\left|t-t^{\prime}\right|$ for all $t, t^{\prime} \in[0, l]$. In particular, $c$ is an isometry and $d(x, y)=l$. The image $\alpha$ of $c$ is called a geodesic (or metric) segment joining $x$ and $y$. When it is unique, this geodesic segment is denoted by $[x, y]$. The space $(X, d)$ is said to be a geodesic space if every two points of $X$ are joined by a geodesic, and $X$ is said to be uniquely geodesic if there is exactly one geodesic joining $x$ and $y$ for each $x, y \in X$. A subset $Y \subseteq X$ is said to be convex if $Y$ includes every geodesic segment joining any two of its points. A geodesic triangle $\triangle\left(x_{1}, x_{2}, x_{3}\right)$ in a geodesic metric space $(X, d)$ consists of three points $x_{1}, x_{2}, x_{3}$ in $X$ (the vertices of $\Delta$ ) and a geodesic segment between each pair of vertices

(c) 2013 Wangkeeree and Preechasilp; licensee Springer. This is an Open Access article distributed under the terms of the Creative Commons Attribution License (http://creativecommons.org/licenses/by/2.0), which permits unrestricted use, distribution, and reproduction in any medium, provided the original work is properly cited. 
(the edges of $\triangle$ ). A comparison triangle for the geodesic triangle $\triangle\left(x_{1}, x_{2}, x_{3}\right)$ in $(X, d)$ is a triangle $\bar{\triangle}\left(x_{1}, x_{2}, x_{3}\right):=\triangle\left(\bar{x}_{1}, \bar{x}_{2}, \bar{x}_{3}\right)$ in the Euclidean plane $\mathbb{E}^{2}$ such that $d_{\mathbb{E}_{2}}\left(\bar{x}_{i}, \bar{x}_{j}\right)=d\left(x_{i}, x_{j}\right)$ for all $i, j \in\{1,2,3\}$.

A geodesic space is said to be a CAT(0) space if all geodesic triangles of an appropriate size satisfy the following comparison axiom.

$\mathrm{CAT}(0)$ : Let $\triangle$ be a geodesic triangle in $X$ and let $\bar{\Delta}$ be a comparison triangle for $\Delta$. Then $\triangle$ is said to satisfy the $\mathrm{CAT}(0)$ inequality if for all $x, y \in \Delta$ and all comparison points $\bar{x}, \bar{y} \in \bar{\triangle}$,

$$
d(x, y) \leq d_{\mathbb{E}^{2}}(\bar{x}, \bar{y})
$$

If $x, y_{1}, y_{2}$ are points in a $\operatorname{CAT}(0)$ space and if $y_{0}$ is the midpoint of the segment $\left[y_{1}, y_{2}\right]$, then the $\operatorname{CAT}(0)$ inequality implies

$$
d^{2}\left(x, y_{0}\right) \leq \frac{1}{2} d^{2}\left(x, y_{1}\right)+\frac{1}{2} d^{2}\left(x, y_{2}\right)-\frac{1}{4} d^{2}\left(y_{1}, y_{2}\right) .
$$

This is the $(\mathrm{CN})$-inequality of Bruhat and Tits [1]. In fact ( $c f$. [2], p.163), a geodesic space is a $\mathrm{CAT}(0)$ space if and only if it satisfies the $(\mathrm{CN})$-inequality.

It is well known that any complete, simply connected Riemannian manifold having nonpositive sectional curvature is a CAT $(0)$ space. Other examples include pre-Hilbert spaces, $\mathbb{R}$-trees (see [2]), Euclidean buildings (see [3]), the complex Hilbert ball with a hyperbolic metric (see [4]), and many others. Complete CAT(0) spaces are often called Hadamard spaces.

It is proved in [2] that a normed linear space satisfies the (CN)-inequality if and only if it satisfies the parallelogram identity, i.e., is a pre-Hilbert space; hence it is not so unusual to have an inner product-like notion in Hadamard spaces. Berg and Nikolaev [5] introduced the concept of quasilinearization as follows:

Let us formally denote a pair $(a, b) \in X \times X$ by $\overrightarrow{a b}$ and call it a vector. Then quasilinearization is defined as a map $\langle\cdot, \cdot\rangle:(X \times X) \times(X \times X) \rightarrow \mathbb{R}$ defined by

$$
\langle\overrightarrow{a b}, \overrightarrow{c d}\rangle=\frac{1}{2}\left(d^{2}(a, d)+d^{2}(b, c)-d^{2}(a, c)-d^{2}(b, d)\right) \quad(a, b, c, d \in X) .
$$

It is easily seen that $\langle\overrightarrow{a b}, \overrightarrow{c d}\rangle=\langle\overrightarrow{c d}, \overrightarrow{a b}\rangle,\langle\overrightarrow{a b}, \overrightarrow{c d}\rangle=-\langle\overrightarrow{b a}, \overrightarrow{c d}\rangle$, and $\langle\overrightarrow{a x}, \overrightarrow{c d}\rangle+\langle\overrightarrow{x b}, \overrightarrow{c d}\rangle=\langle\overrightarrow{a b}, \overrightarrow{c d}\rangle$ for all $a, b, c, d, x \in X$. We say that $X$ satisfies the Cauchy-Schwarz inequality if

$$
\langle\overrightarrow{a b}, \overrightarrow{c d}\rangle \leq d(a, b) d(c, d)
$$

for all $a, b, c, d \in X$. It is known [5, Corollary 3] that a geodesically connected metric space is a $\operatorname{CAT}(0)$ space if and only if it satisfies the Cauchy-Schwarz inequality.

In 2010, Kakavandi and Amini [6] introduced the concept of a dual space for CAT(0) spaces as follows. Consider the map $\Theta: \mathbb{R} \times X \times X \rightarrow C(X)$ defined by

$$
\Theta(t, a, b)(x)=t\langle\overrightarrow{a b}, \overrightarrow{a x}\rangle,
$$

where $C(X)$ is the space of all continuous real-valued functions on $X$. Then the CauchySchwarz inequality implies that $\Theta(t, a, b)$ is a Lipschitz function with the Lipschitz semi- 
norm $L(\Theta(t, a, b))=|t| d(a, b)$ for all $t \in \mathbb{R}$ and $a, b \in X$, where

$$
L(f)=\sup \left\{\frac{f(x)-f(y)}{d(x, y)}: x, y \in X, x \neq y\right\}
$$

is the Lipschitz semi-norm of the function $f: X \rightarrow \mathbb{R}$. Now, define the pseudometric $D$ on $\mathbb{R} \times X \times X$ by

$$
D((t, a, b),(s, c, d))=L(\Theta(t, a, b)-\Theta(s, c, d)) .
$$

Lemma 1.1 [6, Lemma 2.1] $D((t, a, b),(s, c, d))=0$ if and only if $t\langle\overrightarrow{a b}, \overrightarrow{x y}\rangle=s\langle\overrightarrow{c d}, \overrightarrow{x y}\rangle$ for all $x, y \in X$.

For a complete CAT $(0)$ space $(X, d)$, the pseudometric space $(\mathbb{R} \times X \times X, D)$ can be considered as a subspace of the pseudometric space $(\operatorname{Lip}(X, \mathbb{R}), L)$ of all real-valued Lipschitz functions. Also, $D$ defines an equivalence relation on $\mathbb{R} \times X \times X$, where the equivalence class of $\overrightarrow{t a b}:=(t, a, b)$ is

$$
[\overrightarrow{t a b}]=\{s \overrightarrow{s d}: t\langle\overrightarrow{a b}, \overrightarrow{x y}\rangle=s\langle\overrightarrow{c d}, \overrightarrow{x y}\rangle \forall x, y \in X\}
$$

The set $X^{*}:=\{[t \overrightarrow{a b}]:(t, a, b) \in \mathbb{R} \times X \times X\}$ is a metric space with a metric $D$, which is called the dual metric space of $(X, d)$.

Recently, Dehghan and Rooin [7] introduced the duality mapping in CAT(0) spaces and studied its relation with subdifferential by using the concept of quasilinearization. Then they presented a characterization of a metric projection in CAT(0) spaces as follows.

Theorem 1.2 [7, Theorem 2.4] Let $C$ be a nonempty convex subset of a complete CAT(0) space $X, x \in X$ and $u \in C$. Then

$$
u=P_{C} x \quad \text { if and only if } \quad\langle\overrightarrow{y u}, \overrightarrow{u x}\rangle \geq 0 \text { for all } y \in C
$$

Let $C$ be a nonempty subset of a complete $C A T(0)$ space $X$. Then the mapping $T$ of $C$ into itself is called nonexpansive iff $d(T x, T y) \leq d(x, y)$ for all $x, y \in C$. A point $x \in C$ is called a fixed point of $T$ if $x=T x$. We denote by $F(T)$ the set of all fixed points of $T$. Kirk [8] showed that the fixed point set of a nonexpansive mapping $T$ is closed and convex. A mapping $f$ of $C$ into itself is called contraction with coefficient $\alpha \in(0,1)$ iff $d(f x, f y) \leq$ $\alpha d(x, y)$ for all $x, y \in C$. Banach's contraction principle [9] guarantees that $f$ has a unique fixed point when $C$ is a nonempty closed convex subset of a complete metric space. The existence of fixed points and convergence theorems for several mappings in CAT(0) spaces have been investigated by many authors (see also [10-16]).

In 2010, Saejung [15] studied the convergence theorems of the following Halpern's iterations for a nonexpansive mapping $T$ : Let $u$ be fixed and $x_{t} \in C$ be the unique fixed point of the contraction $x \mapsto t u \oplus(1-t) T x$; i.e.,

$$
x_{t}=t u \oplus(1-t) T x_{t},
$$


where $t \in[0,1]$ and $x_{0}, u \in C$ are arbitrarily chosen and

$$
x_{n+1}=\alpha_{n} u \oplus\left(1-\alpha_{n}\right) T x_{n}, \quad n \geq 0,
$$

where $\left\{\alpha_{n}\right\} \subset(0,1)$. It is proved in [15] that $\left\{x_{t}\right\}$ converges strongly as $t \rightarrow 0$ to $\tilde{x} \in F(T)$ which is nearest to $u\left(\tilde{x}=P_{F(T)} u\right)$, and $\left\{x_{n}\right\}$ converges strongly as $n \rightarrow \infty$ to $\tilde{x} \in F(T)$ which is nearest to $u$ under certain appropriate conditions on $\left\{\alpha_{n}\right\}$, where $P_{C} x$ is a metric projection from $X$ onto $C$.

In 2012, Shi and Chen [16] studied the convergence theorems of the following Moudafi's viscosity iterations for a nonexpansive mapping $T$ : For a contraction $f$ on $C$ and $t \in(0,1)$, let $x_{t} \in C$ be the unique fixed point of the contraction $x \mapsto t f(x) \oplus(1-t) T x$; i.e.,

$$
x_{t}=t f\left(x_{t}\right) \oplus(1-t) T x_{t},
$$

and $x_{0} \in C$ is arbitrarily chosen and

$$
x_{n+1}=\alpha_{n} f\left(x_{n}\right) \oplus\left(1-\alpha_{n}\right) T x_{n}, \quad n \geq 0,
$$

where $\left\{\alpha_{n}\right\} \subset(0,1)$. They proved that $\left\{x_{t}\right\}$ defined by (6) converges strongly as $t \rightarrow 0$ to $\tilde{x} \in F(T)$ such that $\tilde{x}=P_{F(T)} f(\tilde{x})$ in the framework of a CAT(0) space satisfying the property $\mathcal{P}$, i.e., if for $x, u, y_{1}, y_{2} \in X$,

$$
d\left(x, P_{\left[x, y_{1}\right]} u\right) d\left(x, y_{1}\right) \leq d\left(x, P_{\left[x, y_{2}\right]} u\right) d\left(x, y_{2}\right)+d(x, u) d\left(y_{1}, y_{2}\right)
$$

Furthermore, they also obtained that $\left\{x_{n}\right\}$ defined by (7) converges strongly as $n \rightarrow \infty$ to $\tilde{x} \in F(T)$ under certain appropriate conditions imposed on $\left\{\alpha_{n}\right\}$.

All of the above bring us the following conjectures.

Question 1.3 Could we obtain the strong convergence of both $\left\{x_{t}\right\}$ and $\left\{x_{n}\right\}$ defined by (6) and (7) respectively, in the framework of a $\mathrm{CAT}(0)$ space without the property $\mathcal{P}$ ?

The purpose of this paper is to study the strong convergence theorems of the iterative schemes (6) and (7) in $\operatorname{CAT}(0)$ spaces without the property $\mathcal{P}$. We prove that the iterative schemes (6) and (7) converge strongly to $\tilde{x}$ such that $\tilde{x}=P_{F(T)} f(\tilde{x})$, which is the unique solution of the variational inequality (VIP)

$$
\langle\overrightarrow{\tilde{x} f} \tilde{x}, \vec{x} \tilde{x}\rangle \geq 0, \quad x \in F(T) .
$$

By using the concept of quasilinearization, we remark that the proof given below is different from that of Shi and Chen [16]. In fact, strong convergence theorems for two given iterative schemes are established in $\operatorname{CAT}(0)$ spaces without the property $\mathcal{P}$.

\section{Preliminaries}

In this paper, we write $(1-t) x \oplus t y$ for the unique point $z$ in the geodesic segment joining from $x$ to $y$ such that

$$
d(z, x)=t d(x, y) \quad \text { and } \quad d(z, y)=(1-t) d(x, y) \text {. }
$$


We also denote by $[x, y]$ the geodesic segment joining from $x$ to $y$, that is, $[x, y]=\{(1-t) x \oplus$ ty $: t \in[0,1]\}$. A subset $C$ of a $C A T(0)$ space is convex if $[x, y] \subseteq C$ for all $x, y \in C$.

The following lemmas play an important role in our paper.

Lemma 2.1 [2, Proposition 2.2] Let $X$ be a $C A T(0)$ space, $p, q, r, s \in X$ and $\lambda \in[0,1]$. Then

$$
d(\lambda p \oplus(1-\lambda) q, \lambda r \oplus(1-\lambda) s) \leq \lambda d(p, r)+(1-\lambda) d(q, s) .
$$

Lemma 2.2 [11, Lemma 2.4] Let $X$ be a CAT(0) space, $x, y, z \in X$ and $\lambda \in[0,1]$. Then

$$
d(\lambda x \oplus(1-\lambda) y, z) \leq \lambda d(x, z)+(1-\lambda) d(y, z) .
$$

Lemma 2.3 [11, Lemma 2.5] Let $X$ be a CAT(0) space, $x, y, z \in X$ and $\lambda \in[0,1]$. Then

$$
d^{2}(\lambda x \oplus(1-\lambda) y, z) \leq \lambda d^{2}(x, z)+(1-\lambda) d^{2}(y, z)-\lambda(1-\lambda) d^{2}(x, y) .
$$

The concept of $\Delta$-convergence introduced by Lim [17] in 1976 was shown by Kirk and Panyanak [18] in CAT(0) spaces to be very similar to weak convergence in the Banach space setting. Next, we give the concept of $\Delta$-convergence and collect some basic properties.

Let $\left\{x_{n}\right\}$ be a bounded sequence in a $\operatorname{CAT}(0)$ space $X$. For $x \in X$, we set

$$
r\left(x,\left\{x_{n}\right\}\right)=\limsup _{n \rightarrow \infty} d\left(x, x_{n}\right)
$$

The asymptotic radius $r\left(\left\{x_{n}\right\}\right)$ of $\left\{x_{n}\right\}$ is given by

$$
r\left(\left\{x_{n}\right\}\right)=\inf \left\{r\left(x,\left\{x_{n}\right\}\right): x \in X\right\},
$$

and the asymptotic center $A\left(\left\{x_{n}\right\}\right)$ of $\left\{x_{n}\right\}$ is the set

$$
A\left(\left\{x_{n}\right\}\right)=\left\{x \in X: r\left(x,\left\{x_{n}\right\}\right)=r\left(\left\{x_{n}\right\}\right)\right\} .
$$

It is known from Proposition 7 of [14] that in a CAT $(0)$ space, $A\left(\left\{x_{n}\right\}\right)$ consists of exactly one point. A sequence $\left\{x_{n}\right\} \subset X$ is said to $\Delta$-converge to $x \in X$ if $A\left(\left\{x_{n_{k}}\right\}\right)=\{x\}$ for every subsequence $\left\{x_{n_{k}}\right\}$ of $\left\{x_{n}\right\}$. The uniqueness of an asymptotic center implies that the CAT( 0$)$ space $X$ satisfies Opial's property, i.e., for given $\left\{x_{n}\right\} \subset X$ such that $\left\{x_{n}\right\} \Delta$-converges to $x$ and given $y \in X$ with $y \neq x$,

$$
\limsup _{n \rightarrow \infty} d\left(x_{n}, x\right)<\limsup _{n \rightarrow \infty} d\left(x_{n}, y\right)
$$

Since it is not possible to formulate the concept of demiclosedness in a CAT(0) setting, as stated in linear spaces, let us formally say that ' $I-T$ is demiclosed at zero' if the conditions $\left\{x_{n}\right\} \subseteq C \Delta$-converges to $x$ and $d\left(x_{n}, T x_{n}\right) \rightarrow 0$ imply $x \in F(T)$.

Lemma 2.4 [18] Every bounded sequence in a complete CAT(0) space always has a $\Delta$ convergent subsequence. 
Lemma 2.5 [13] If $C$ is a closed convex subset of a complete $C A T(0)$ space and if $\left\{x_{n}\right\}$ is a bounded sequence in $C$, then the asymptotic center of $\left\{x_{n}\right\}$ is in $C$.

Lemma 2.6 [13] If $C$ is a closed convex subset of $X$ and $T: C \rightarrow X$ is a nonexpansive mapping, then the conditions $\left\{x_{n}\right\} \Delta$-converges to $x$ and $d\left(x_{n}, T x_{n}\right) \rightarrow 0$ imply $x \in C$ and $T x=x$.

Having the notion of quasilinearization, Kakavandi and Amini [6] introduced the following notion of convergence.

A sequence $\left\{x_{n}\right\}$ in the complete $\operatorname{CAT}(0)$ space $(X, d) w$-converges to $x \in X$ if $\lim _{n \rightarrow \infty}\left\langle\overrightarrow{x x_{n}}, \overrightarrow{x y}\right\rangle=0$, i.e., $\lim _{n \rightarrow \infty}\left(d^{2}\left(x_{n}, x\right)-d^{2}\left(x_{n}, y\right)+d^{2}(x, y)\right)=0$ for all $y \in X$.

It is obvious that convergence in the metric implies $w$-convergence, and it is easy to check that $w$-convergence implies $\Delta$-convergence [6, Proposition 2.5], but it is showed in ([19, Example 4.7]) that the converse is not valid. However, the following lemma shows another characterization of $\Delta$-convergence as well as, more explicitly, a relation between $w$-convergence and $\Delta$-convergence.

Lemma 2.7 [19, Theorem 2.6] Let $X$ be a complete CAT( 0$)$ space, $\left\{x_{n}\right\}$ be a sequence in $X$, and $x \in X$. Then $\left\{x_{n}\right\} \Delta$-converges to $x$ if and only if $\lim \sup _{n \rightarrow \infty}\left\langle\overrightarrow{x x_{n}}, \overrightarrow{x y}\right\rangle \leq 0$ for all $y \in X$.

Lemma 2.8 [20, Lemma 2.1] Let $\left\{a_{n}\right\}$ be a sequence of non-negative real numbers satisfying the property

$$
a_{n+1} \leq\left(1-\alpha_{n}\right) a_{n}+\alpha_{n} \beta_{n}, \quad n \geq 0,
$$

where $\left\{\alpha_{n}\right\} \subseteq(0,1)$ and $\left\{\beta_{n}\right\} \subseteq \mathbb{R}$ such that

1. $\sum_{n=0}^{\infty} \alpha_{n}=\infty$;

2. $\limsup _{n \rightarrow \infty} \beta_{n} \leq 0$ or $\sum_{n=0}^{\infty}\left|\alpha_{n} \beta_{n}\right|<\infty$.

Then $\left\{a_{n}\right\}$ converges to zero as $n \rightarrow \infty$.

The following two lemmas can be obtained from elementary computation. For convenience of the readers, we include the details.

Lemma 2.9 Let $X$ be a complete $C A T(0)$ space. Then for all $u, x, y \in X$, the following inequality holds:

$$
d^{2}(x, u) \leq d^{2}(y, u)+2\langle\overrightarrow{x y}, \overrightarrow{x u}\rangle .
$$

Proof

$$
\begin{aligned}
d^{2}(y, u)-d^{2}(x, u)-2\langle\overrightarrow{y x}, \overrightarrow{x u}\rangle & =d^{2}(y, u)-d^{2}(x, u)-2\langle\overrightarrow{y u}, \overrightarrow{x u}\rangle-2\langle\overrightarrow{u x}, \overrightarrow{x u}\rangle \\
& =d^{2}(y, u)-d^{2}(x, u)-2\langle\overrightarrow{y u}, \overrightarrow{x u}\rangle+2 d^{2}(x, u) \\
& =d^{2}(y, u)+d^{2}(x, u)-2\langle\overrightarrow{y u}, \overrightarrow{x u}\rangle \\
& \geq d^{2}(y, u)+d^{2}(x, u)-2 d(y, u) d(x, u) \\
& =(d(y, u)-d(x, u))^{2} \geq 0 .
\end{aligned}
$$


Hence

$$
d^{2}(x, u) \leq d^{2}(y, u)+2\langle\overrightarrow{x y}, \overrightarrow{x u}\rangle
$$

Lemma 2.10 Let $X$ be a CAT(0) space. For any $u, v \in X$ and $t \in[0,1]$, let $u_{t}=t u \oplus(1-t) v$. Then, for all $x, y \in X$,

(i) $\left\langle\overrightarrow{u_{t} x}, \overrightarrow{u_{t} y}\right\rangle \leq t\left\langle\overrightarrow{u x}, \overrightarrow{u_{t} y}\right\rangle+(1-t)\left\langle\overrightarrow{v x}, \overrightarrow{u_{t} y}\right\rangle$;

(ii) $\left\langle\overrightarrow{u_{t} x}, \overrightarrow{u y}\right\rangle \leq t\langle\overrightarrow{u x}, \overrightarrow{u y}\rangle+(1-t)\langle\overrightarrow{v x}, \overrightarrow{u y}\rangle$ and $\left\langle\overrightarrow{u_{t} x}, \overrightarrow{v y}\right\rangle \leq t\langle\overrightarrow{u x}, \overrightarrow{v y}\rangle+(1-t)\langle\overrightarrow{v x}, \overrightarrow{v y}\rangle$.

Proof (i) It follows from the (CN)-inequality that

$$
\begin{aligned}
2\left\langle\overrightarrow{u_{t} x}, \overrightarrow{u_{t} y}\right\rangle= & d^{2}\left(u_{t}, y\right)+d^{2}\left(x, u_{t}\right)-d^{2}\left(u_{t}, u_{t}\right)-d^{2}(x, y) \\
\leq & t d^{2}(u, y)+(1-t) d^{2}(v, y)-t(1-t) d^{2}(u, v)+d^{2}\left(x, u_{t}\right) \\
& -d^{2}\left(u_{t}, u_{t}\right)-d^{2}(x, y) \\
= & t d^{2}(u, y)+t d^{2}\left(x, u_{t}\right)-t d^{2}\left(u, u_{t}\right)-t d^{2}(x, y) \\
& +(1-t) d^{2}(v, y)+(1-t) d^{2}\left(x, u_{t}\right)-(1-t) d^{2}\left(v, u_{t}\right)-(1-t) d^{2}(x, y) \\
& +t d^{2}\left(u, u_{t}\right)+(1-t) d^{2}\left(v, u_{t}\right)-t(1-t) d^{2}(u, v) \\
= & t\left[d^{2}(u, y)+d^{2}\left(x, u_{t}\right)-d^{2}\left(u, u_{t}\right)-d^{2}(x, y)\right] \\
& +(1-t)\left[d^{2}(v, y)+d^{2}\left(x, u_{t}\right)-d^{2}\left(v, u_{t}\right)-d^{2}(x, y)\right] \\
& +t(1-t)^{2} d^{2}(u, v)+(1-t) t^{2} d^{2}(u, v)-t(1-t) d^{2}(u, v) \\
= & t\left\langle\overrightarrow{u x}, \overrightarrow{u_{t} y}\right\rangle+(1-t)\left\langle\overrightarrow{v x}, \overrightarrow{u_{t} y}\right\rangle .
\end{aligned}
$$

(ii) The proof is similar to (i).

\section{Variational inequalities in CAT(0) spaces}

In this section, we present strong convergence theorems of Moudafi's viscosity methods in CAT(0) spaces. Our first result is the continuous version of Theorem 2.2 of Shi and Chen [16]. By using the concept of quasilinearization, we note that the proof given below is different from that of Shi and Chen.

For any $t \in(0,1]$ and a contraction $f$ with coefficient $\alpha \in(0,1)$, define the mapping $S_{t}$ : $C \rightarrow C$ by

$$
G_{t}=t f(x) \oplus(1-t) T x, \quad \forall x \in C
$$

It is not hard to see that $G_{t}$ is a contraction on $C$. Indeed, for $x, y \in C$, we have

$$
\begin{aligned}
& d\left(G_{t}(x), G_{t}(y)\right) \\
& =d(t f(x) \oplus(1-t) T x, t f(y) \oplus(1-t) T y) \\
& \leq d(t f(x) \oplus(1-t) T x, t f(y) \oplus(1-t) T x) \\
& \quad+d(t f(y) \oplus(1-t) T x, t f(y) \oplus(1-t) T y) \\
& \leq t d(f(x), f(y))+(1-t) d(T x, T y)
\end{aligned}
$$




$$
\begin{aligned}
& \leq t \alpha d(x, y)+(1-t) d(x, y) \\
& =(1-t(1-\alpha)) d(x, y)
\end{aligned}
$$

This implies that $G_{t}$ is a contraction mapping. Then there exists a unique $u \in C$ such that

$$
u=G_{t}(u)=t f(u) \oplus(1-t) T u .
$$

Let $x_{t} \in C$ be the unique fixed point of $G_{t}$. Thus

$$
x_{t}=t f\left(x_{t}\right) \oplus(1-t) T x_{t} .
$$

Theorem 3.1 Let $C$ be a closed convex subset of a complete CAT(0) space $X$, and let $T: C \rightarrow C$ be a nonexpansive mapping with $F(T) \neq \emptyset$. Let $f$ be a contraction on $C$ with coefficient $0<\alpha<1$. For each $t \in(0,1]$, let $\left\{x_{t}\right\}$ be given by

$$
x_{t}=t f\left(x_{t}\right) \oplus(1-t) T x_{t} .
$$

Then $\left\{x_{t}\right\}$ converges strongly as $t \rightarrow 0$ to $\tilde{x}$ such that $\tilde{x}=P_{F(T)} f(\tilde{x})$ which is equivalent to the following variational inequality:

$$
\langle\overrightarrow{\tilde{x} f(\tilde{x})}, \vec{x} \tilde{x}\rangle \geq 0, \quad x \in F(T)
$$

Proof We first show that $\left\{x_{t}\right\}$ is bounded. For any $p \in F(T)$, we have that

$$
\begin{aligned}
d\left(x_{t}, p\right) & =d\left(t f\left(x_{t}\right) \oplus(1-t) T x_{t}, p\right) \\
& \leq t d\left(f\left(x_{t}\right), p\right)+(1-t) d\left(T x_{t}, p\right) \\
& \leq t d\left(f\left(x_{t}\right), p\right)+(1-t) d\left(x_{t}, p\right) .
\end{aligned}
$$

Then

$$
\begin{aligned}
d\left(x_{t}, p\right) & \leq d\left(f\left(x_{t}\right), p\right) \leq d\left(f\left(x_{t}\right), f(p)\right)+d(f(p), p) \\
& \leq \alpha d\left(x_{t}, p\right)+d(f(p), p) .
\end{aligned}
$$

This implies that

$$
d\left(x_{t}, p\right) \leq \frac{1}{1-\alpha} d(f(p), p)
$$

Hence $\left\{x_{t}\right\}$ is bounded, so are $\left\{T x_{t}\right\}$ and $\left\{f\left(x_{t}\right)\right\}$. We get that

$$
\begin{aligned}
\lim _{t \rightarrow 0} d\left(x_{t}, T x_{t}\right) & =\lim _{t \rightarrow 0} d\left(t f\left(x_{t}\right) \oplus(1-t) T x_{t}, T x_{t}\right) \\
& \leq \lim _{t \rightarrow 0}\left[t d\left(f\left(x_{t}\right), T x_{t}\right)+(1-t) d\left(T x_{t}, T x_{t}\right)\right] \\
& \leq \lim _{t \rightarrow 0} t d\left(f\left(x_{t}\right), T x_{t}\right)=0 .
\end{aligned}
$$


Assume that $\left\{t_{n}\right\} \subset(0,1)$ is such that $t_{n} \rightarrow 0$ as $n \rightarrow \infty$. Put $x_{n}:=x_{t_{n}}$. We will show that $\left\{x_{n}\right\}$ contains a subsequence converging strongly to $\tilde{x}$ such that $\tilde{x}=P_{F(T)} f(\tilde{x})$ which is equivalent to the following variational inequality:

$$
|\overrightarrow{\tilde{x} f(\tilde{x})}, \overrightarrow{x \tilde{x}}\rangle \geq 0, \quad x \in F(T) .
$$

Since $\left\{x_{n}\right\}$ is bounded, by Lemma $2.4,2.6$, we may assume that $\left\{x_{n}\right\} \Delta$-converges to a point $\tilde{x}$ and $\tilde{x} \in F(T)$. It follows from Lemma 2.10 (i) that

$$
\begin{aligned}
d^{2}\left(x_{n}, \tilde{x}\right) & =\left\langle\overrightarrow{x_{n} \tilde{x}}, \overrightarrow{x_{n}} \vec{x}\right\rangle \\
& \leq \alpha_{n}\left|\overrightarrow{f\left(x_{n}\right) \tilde{x}}, \overrightarrow{x_{n}} \overrightarrow{\tilde{x}}\right\rangle+\left(1-\alpha_{n}\right)\left\langle\overrightarrow{T x_{n}} \overrightarrow{\tilde{x}}, \overrightarrow{x_{n}} \overrightarrow{\tilde{x}}\right\rangle \\
& \leq \alpha_{n}\left|\overrightarrow{f\left(x_{n}\right) \tilde{x}}, \overrightarrow{x_{n} \tilde{x}}\right|+\left(1-\alpha_{n}\right) d\left(T x_{n}, \tilde{x}\right) d\left(x_{n}, \tilde{x}\right) \\
& \leq \alpha_{n}\left|\overrightarrow{f\left(x_{n}\right) \tilde{x}}, \overrightarrow{x_{n}} \overrightarrow{\tilde{x}}\right|+\left(1-\alpha_{n}\right) d^{2}\left(x_{n}, \tilde{x}\right) .
\end{aligned}
$$

It follows that

$$
\begin{aligned}
& d^{2}\left(x_{n}, \tilde{x}\right) \leq\left\langle\overrightarrow{f\left(x_{n}\right) \tilde{x}}, \overrightarrow{x_{n}} \overrightarrow{\tilde{x}}\right\rangle \\
& =\left\langle\overrightarrow{f\left(x_{n}\right) f(\tilde{x})}, \overrightarrow{x_{n}} \overrightarrow{\tilde{x}}\right\rangle+\left\langle\overrightarrow{f(\tilde{x}) \tilde{x}}, \overrightarrow{x_{n}} \overrightarrow{\tilde{x}}\right\rangle \\
& \leq d\left(f\left(x_{n}\right), f(\tilde{x})\right) d\left(x_{n}, \tilde{x}\right)+\left\langle\overrightarrow{f(\tilde{x}) \tilde{x}}, \overrightarrow{x_{n} \tilde{x}}\right\rangle \\
& \leq \alpha d^{2}\left(x_{n}, \tilde{x}\right)+\left\langle\overrightarrow{f(\tilde{x}) \tilde{x}}, \overrightarrow{x_{n}} \overrightarrow{\tilde{x}}\right\rangle,
\end{aligned}
$$

and thus

$$
d^{2}\left(x_{n}, \tilde{x}\right) \leq \frac{1}{1-\alpha}\left\langle\overrightarrow{f(\tilde{x}) \tilde{x}}, \overrightarrow{x_{n}} \vec{x}\right| .
$$

Since $\left\{x_{n}\right\} \Delta$-converges to $\tilde{x}$, by Lemma 2.7 , we have

$$
\limsup _{n \rightarrow \infty}\left|\overrightarrow{f(\tilde{x}) \tilde{x}}, \overrightarrow{x_{n}} \vec{x}\right\rangle \leq 0
$$

It follows from (13) that $\left\{x_{n}\right\}$ converges strongly to $\tilde{x}$.

Next, we show that $\tilde{x}$ solves the variational inequality (12). Applying Lemma 2.3, for any $q \in F(T)$,

$$
\begin{aligned}
d^{2}\left(x_{t}, q\right) & =d^{2}\left(t f\left(x_{t}\right) \oplus(1-t) T x_{t}, q\right) \\
& \leq t d^{2}\left(f\left(x_{t}\right), q\right)+(1-t) d^{2}\left(T x_{t}, q\right)-t(1-t) d^{2}\left(f\left(x_{t}\right), T x_{t}\right) \\
& \leq t d^{2}\left(f\left(x_{t}\right), q\right)+(1-t) d^{2}\left(x_{t}, q\right)-t(1-t) d^{2}\left(f\left(x_{t}\right), T x_{t}\right) .
\end{aligned}
$$

It implies that

$$
d^{2}\left(x_{t}, q\right) \leq d^{2}\left(f\left(x_{t}\right), q\right)-(1-t) d^{2}\left(f\left(x_{t}\right), T x_{t}\right) .
$$

Taking the limit through $t=t_{n} \rightarrow 0$, we can get that

$$
d^{2}(\tilde{x}, q) \leq d^{2}(f(\tilde{x}), q)-d^{2}(f(\tilde{x}), \tilde{x}) .
$$


Hence

$$
0 \leq \frac{1}{2}\left[d^{2}(\tilde{x}, \tilde{x})+d^{2}(f(\tilde{x}), q)-d^{2}(\tilde{x}, q)-d^{2}(f(\tilde{x}), \tilde{x})\right]=\langle\overrightarrow{\tilde{x} f(\tilde{x})}, \overrightarrow{q \tilde{x}}\rangle, \quad \forall q \in F(T)
$$

That is, $\tilde{x}$ solves the inequality (12).

Finally, we show that the entire net $\left\{x_{t}\right\}$ converges to $\tilde{x}$, assume $x_{s_{n}} \rightarrow \hat{x}$, where $s_{n} \rightarrow 0$. By the same argument, we get that $\hat{x} \in F(T)$ and solves the variational inequality (12), i.e.,

$$
\langle\overrightarrow{\tilde{x} f(\tilde{x})}, \overrightarrow{\tilde{x} \hat{x}}\rangle \leq 0
$$

and

$$
\langle\overrightarrow{\hat{x} f(\hat{x}), \overrightarrow{\hat{x}} \tilde{x}}\rangle \leq 0
$$

Adding up (15) and (16), we get that

$$
\begin{aligned}
0 & \geq\langle\overrightarrow{\tilde{x} f(\tilde{x})}, \overrightarrow{\tilde{x} \hat{x}}\rangle-\langle\overrightarrow{\hat{x} f(\hat{x}), \vec{x} \hat{x}}\rangle \\
& =\langle\overrightarrow{\tilde{x} f(\hat{x}), \vec{x} \hat{x}}\rangle+\langle\overrightarrow{f(\hat{x}) f(\tilde{x}),}, \overrightarrow{\tilde{x} \hat{x}}\rangle-\langle\overrightarrow{\hat{x} \tilde{x}}, \overrightarrow{\tilde{x} \hat{x}}\rangle-\langle\overrightarrow{\tilde{x} f(\hat{x}), \overrightarrow{\tilde{x} \hat{x}}\rangle} \\
& =\langle\overrightarrow{\tilde{x} \hat{x}}, \overrightarrow{\tilde{x} \hat{x}}\rangle-\langle\overrightarrow{f(\hat{x}) f(\vec{x}), \overrightarrow{\hat{x}} \tilde{x}\rangle} \\
& \geq\langle\overrightarrow{\tilde{x} \hat{x}}, \overrightarrow{\tilde{x} \hat{x}}\rangle-d(f(\hat{x}), f(\tilde{x})) d(\hat{x}, \tilde{x}) \\
& \geq d^{2}(\tilde{x}, \hat{x})-\alpha d(\hat{x}, \tilde{x}) d(\hat{x}, \tilde{x}) \\
& =d^{2}(\tilde{x}, \hat{x})-\alpha d^{2}(\hat{x}, \tilde{x}) \\
& \geq(1-\alpha) d^{2}(\tilde{x}, \hat{x}) .
\end{aligned}
$$

Since $0<\alpha<1$, we have that $d(\tilde{x}, \hat{x})=0$, and so $\tilde{x}=\hat{x}$. Hence the net $x_{t}$ converges strongly to $\tilde{x}$ which is the unique solution to the variational inequality (12). This completes the proof.

Remark 3.2 We give the different proof of [16, Theorem 2.2]. In fact, the property $\mathcal{P}$ imposed on a $\operatorname{CAT}(0)$ space $X$ is removed.

If $f \equiv u$, then the following result can be obtained directly from Theorem 3.1.

Corollary 3.3 [15, Lemma 2.2] Let $C$ be a closed convex subset of a complete CAT(0) space $X$, and let $T: C \rightarrow C$ be a nonexpansive mapping with $F(T) \neq \emptyset$. For each $t \in(0,1]$, let $u$ be fixed and $\left\{x_{t}\right\}$ be given by

$$
x_{t}=t u \oplus(1-t) T x_{t} .
$$

Then $\left\{x_{t}\right\}$ converges strongly as $t \rightarrow 0$ to $\tilde{x} \in F(T)$ which is nearest to $u$ which is equivalent to the following variational inequality:

$$
\langle\overrightarrow{\tilde{x} u}, \vec{x} \tilde{x}\rangle \geq 0, \quad x \in F(T) .
$$


Theorem 3.4 Let $C$ be a closed convex subset of a complete $C A T(0)$ space $X$, and let $T: C \rightarrow C$ be a nonexpansive mapping with $F(T) \neq \emptyset$. Let $f$ be a contraction on $C$ with coefficient $0<\alpha<1$. For the arbitrary initial point $x_{0} \in C$, let $\left\{x_{n}\right\}$ be generated by

$$
x_{n+1}=\alpha_{n} f\left(x_{n}\right) \oplus\left(1-\alpha_{n}\right) T x_{n}, \quad \forall n \geq 0,
$$

where $\left\{\alpha_{n}\right\} \subset(0,1)$ satisfies the following conditions:

(i) $\lim _{n \rightarrow \infty} \alpha_{n}=0$;

(ii) $\sum_{n=0}^{\infty} \alpha_{n}=\infty$;

(iii) either $\sum_{n=0}^{\infty}\left|\alpha_{n+1}-\alpha_{n}\right|<\infty$ or $\lim _{n \rightarrow \infty}\left(\alpha_{n+1} / \alpha_{n}\right)=1$.

Then $\left\{x_{n}\right\}$ converges strongly as $n \rightarrow \infty$ to $\tilde{x}$ such that $\tilde{x}=P_{F(T)} f(\tilde{x})$ which is equivalent to the variational inequality (12).

Proof We first show that the sequence $\left\{x_{n}\right\}$ is bounded. For any $p \in F(T)$, we have that

$$
\begin{aligned}
d\left(x_{n+1}, p\right) & =d\left(\alpha_{n} f\left(x_{n}\right) \oplus\left(1-\alpha_{n}\right) T x_{n}, p\right) \\
& \leq \alpha_{n} d\left(f\left(x_{n}\right), p\right)+\left(1-\alpha_{n}\right) d\left(T x_{n}, p\right) \\
& \leq \alpha_{n}\left(d\left(f\left(x_{n}\right), f(p)\right)+d(f(p), p)\right)+\left(1-\alpha_{n}\right) d\left(T x_{n}, p\right) \\
& \leq \max \left\{d\left(x_{n}, p\right), \frac{1}{1-\alpha} d(f(p), p)\right\} .
\end{aligned}
$$

By induction, we have

$$
d\left(x_{n}, p\right) \leq \max \left\{d\left(x_{0}, p\right), \frac{1}{1-\alpha} d(f(p), p)\right\},
$$

for all $n \in \mathbb{N}$. Hence $\left\{x_{n}\right\}$ is bounded, so are $\left\{T x_{n}\right\}$ and $\left\{f\left(x_{n}\right)\right\}$. Next, we claim that $\lim _{n \rightarrow \infty} d\left(x_{n+1}, x_{n}\right)=0$. To this end, we observe that

$$
\begin{aligned}
d\left(x_{n+1}, x_{n}\right)= & d\left(\alpha_{n} f\left(x_{n}\right) \oplus\left(1-\alpha_{n}\right) T x_{n}, \alpha_{n-1} f\left(x_{n-1}\right) \oplus\left(1-\alpha_{n-1}\right) T x_{n-1}\right) \\
\leq & d\left(\alpha_{n} f\left(x_{n}\right) \oplus\left(1-\alpha_{n}\right) T x_{n}, \alpha_{n} f\left(x_{n}\right) \oplus\left(1-\alpha_{n}\right) T x_{n-1}\right) \\
& +d\left(\alpha_{n} f\left(x_{n}\right) \oplus\left(1-\alpha_{n}\right) T x_{n-1}, \alpha_{n} f\left(x_{n-1}\right) \oplus\left(1-\alpha_{n}\right) T x_{n-1}\right) \\
& +d\left(\alpha_{n} f\left(x_{n-1}\right) \oplus\left(1-\alpha_{n}\right) T x_{n-1}, \alpha_{n-1} f\left(x_{n-1}\right) \oplus\left(1-\alpha_{n-1}\right) T x_{n-1}\right) \\
\leq & \left(1-\alpha_{n}\right) d\left(T x_{n}, T x_{n-1}\right)+\alpha_{n} d\left(f\left(x_{n}\right), f\left(x_{n-1}\right)\right) \\
& +\left|\alpha_{n}-\alpha_{n-1}\right| d\left(f\left(x_{n-1}\right), T x_{n-1}\right) \\
\leq & \left(1-\alpha_{n}\right) d\left(x_{n}, x_{n-1}\right)+\alpha_{n} d\left(f\left(x_{n}\right), f\left(x_{n-1}\right)\right)+\left|\alpha_{n}-\alpha_{n-1}\right| d\left(f\left(x_{n-1}\right), T x_{n-1}\right) \\
\leq & \left(1-\alpha_{n}\right) d\left(x_{n}, x_{n-1}\right)+\alpha_{n} \alpha d\left(x_{n}, x_{n-1}\right)+\left|\alpha_{n}-\alpha_{n-1}\right| d\left(f\left(x_{n-1}\right), T x_{n-1}\right) \\
= & \left(1-\alpha_{n}(1-\alpha)\right) d\left(x_{n}, x_{n-1}\right)+\left|\alpha_{n}-\alpha_{n-1}\right| d\left(f\left(x_{n-1}\right), T x_{n-1}\right) .
\end{aligned}
$$

By the conditions (ii) and (iii) and Lemma 2.8, we have

$$
\lim _{n \rightarrow \infty} d\left(x_{n+1}, x_{n}\right)=0
$$


It follows from (20) and condition (i) that

$$
\begin{aligned}
d\left(x_{n}, T x_{n}\right) & \leq d\left(x_{n}, x_{n+1}\right)+d\left(x_{n+1}, T x_{n}\right) \\
& =d\left(x_{n}, x_{n+1}\right)+d\left(\alpha_{n} f\left(x_{n}\right) \oplus\left(1-\alpha_{n}\right) T x_{n}, T x_{n}\right) \\
& \leq d\left(x_{n}, x_{n+1}\right)+\alpha_{n} d\left(f\left(x_{n}\right), T x_{n}\right) \rightarrow 0 \quad \text { as } n \rightarrow \infty .
\end{aligned}
$$

Let $\left\{x_{t}\right\}$ be a net in $C$ such that

$$
x_{t}=t f\left(x_{t}\right) \oplus(1-t) T x_{t} .
$$

By Theorem 3.1, we have that $\left\{x_{t}\right\}$ converges strongly as $t \rightarrow 0$ to a fixed point $\tilde{x} \in F(T)$, which solves the variational inequality (12). Now, we claim that

$$
\limsup _{n \rightarrow \infty}\left\langle\overrightarrow{f(\tilde{x}) \tilde{x}}, \overrightarrow{x_{n}} \overrightarrow{\tilde{x}}\right\rangle \leq 0
$$

It follows from Lemma 2.10 (i) that

$$
\begin{aligned}
& d^{2}\left(x_{t}, x_{n}\right)=\left\langle\overrightarrow{x_{t} x_{n}}, \overrightarrow{x_{t} x_{n}}\right\rangle \\
& \leq t\left|\overrightarrow{f\left(x_{t}\right) x_{n}}, \overrightarrow{x_{t} x_{n}}\right\rangle+(1-t)\left\langle\overrightarrow{T x_{t} x_{n}}, \overrightarrow{x_{t} x_{n}}\right\rangle \\
& =t\left|\overrightarrow{f\left(x_{t}\right) f(\vec{x})}, \overrightarrow{x_{t} x_{n}}\right\rangle+t\left|\overrightarrow{f(\tilde{x})} \overrightarrow{\tilde{x}}, \overrightarrow{x_{t} x_{n}}\right\rangle+t\left\langle\overrightarrow{\tilde{x} x_{t}}, \overrightarrow{x_{t} x_{n}}\right\rangle+t\left\langle\overrightarrow{x_{t} x_{n}}, \overrightarrow{x_{t} x_{n}}\right\rangle \\
& +(1-t)\left\langle\overrightarrow{T x_{t} T x_{n}}, \overrightarrow{x_{t} x_{n}}\right\rangle+(1-t)\left\langle\overrightarrow{T x_{n} x_{n}}, \overrightarrow{x_{t} x_{n}}\right\rangle \\
& \leq t \alpha d\left(x_{t}, \tilde{x}\right) d\left(x_{t}, x_{n}\right)+t\left|\overrightarrow{f(\tilde{x}) \tilde{x}}, \overrightarrow{x_{t} x_{n}}\right\rangle+t d\left(\tilde{x}, x_{t}\right) d\left(x_{t}, x_{n}\right)+t d^{2}\left(x_{t}, x_{n}\right) \\
& +(1-t) d^{2}\left(x_{t}, x_{n}\right)+(1-t) d\left(T x_{n}, x_{n}\right) d\left(x_{t}, x_{n}\right) \\
& \leq t \alpha d\left(x_{t}, \tilde{x}\right) M+t\left|\overrightarrow{f(\tilde{x}) \tilde{x}}, \overrightarrow{x_{t} x_{n}}\right\rangle+t d\left(\tilde{x}, x_{t}\right) M+t d^{2}\left(x_{t}, x_{n}\right) \\
& +(1-t) d^{2}\left(x_{t}, x_{n}\right)+(1-t) d\left(T x_{n}, x_{n}\right) M \\
& \leq d^{2}\left(x_{t}, x_{n}\right)+t \alpha d\left(x_{t}, \tilde{x}\right) M+t d\left(\tilde{x}, x_{t}\right) M+d\left(T x_{n}, x_{n}\right) M+t\left(\overrightarrow{f(\tilde{x}) \tilde{x}}, \overrightarrow{x_{t} x_{n}}\right),
\end{aligned}
$$

where $M \geq \sup _{m, n \geq 1}\left\{d\left(x_{t}, x_{n}\right)\right\}$. This implies that

$$
\left\langle\overrightarrow{f(\tilde{x}) \tilde{x}}, \overrightarrow{x_{n} x_{t}}\right\rangle \leq(1+\alpha) d\left(x_{t}, \tilde{x}\right) M+\frac{d\left(T x_{n}, x_{n}\right)}{t} M
$$

Taking the limit as $n \rightarrow \infty$ first and then $t \rightarrow 0$, the inequality (22) yields

$$
\limsup _{t \rightarrow 0} \limsup _{n \rightarrow \infty}\left\langle\overrightarrow{f(\tilde{x}) \vec{x}}, \overrightarrow{x_{n} x_{t}}\right\rangle \leq 0
$$

Since $x_{t} \rightarrow \tilde{x}$ as $t \rightarrow 0$ and by the continuity of a metric distance $d$, we have, for any fixed $n \geq 0$,

$$
\begin{aligned}
& \lim _{t \rightarrow 0}\left\langle\overrightarrow{f(\tilde{x}) \tilde{x}}, \overrightarrow{x_{n} x_{t}}\right\rangle \\
& \quad=\lim _{t \rightarrow 0} \frac{1}{2}\left[d^{2}\left(f(\tilde{x}), x_{t}\right)+d^{2}\left(\tilde{x}, x_{n}\right)-d^{2}\left(f(\tilde{x}), x_{n}\right)-d^{2}\left(\tilde{x}, x_{t}\right)\right]
\end{aligned}
$$




$$
\begin{aligned}
& =\frac{1}{2}\left[d^{2}(f(\tilde{x}), \tilde{x})+d^{2}\left(\tilde{x}, x_{n}\right)-d^{2}\left(f(\tilde{x}), x_{n}\right)-d^{2}(\tilde{x}, \tilde{x})\right] \\
& =\left\langle\overrightarrow{f(\tilde{x}) \tilde{x}}, \overrightarrow{x_{n}} \vec{x}\right| .
\end{aligned}
$$

It implies that, for any $\varepsilon>0$, there exists a $\delta>0$ such that

$$
\left\langle\overrightarrow{f(\tilde{x})} \overrightarrow{\tilde{x}}, \overrightarrow{x_{n}} \overrightarrow{\tilde{x}}\right\rangle<\left\langle\overrightarrow{f(\tilde{x})} \overrightarrow{\tilde{x}}, \overrightarrow{x_{n} x_{t}}\right\rangle+\varepsilon, \quad \forall t \in(0, \delta) .
$$

Thus, by the upper limit as $n \rightarrow \infty$ first and then $t \rightarrow 0$, the inequality in (23), we get that

$$
\limsup _{n \rightarrow \infty}\left\langle\overrightarrow{f(\tilde{x})} \vec{x}, \overrightarrow{x_{n}} \overrightarrow{\tilde{x}}\right\rangle \leq \varepsilon
$$

Since $\varepsilon$ is arbitrary, it follows that

$$
\limsup _{n \rightarrow \infty}\left|\overrightarrow{f(\tilde{x}) \tilde{x}}, \overrightarrow{x_{n}} \overrightarrow{\tilde{x}}\right\rangle \leq 0
$$

Finally, we prove that $x_{n} \rightarrow \tilde{x}$ as $n \rightarrow \infty$. For any $n \in \mathbb{N}$, we set $y_{n}=\alpha_{n} \tilde{x} \oplus\left(1-\alpha_{n}\right) T x_{n}$. It follows from Lemma 2.9 and Lemma 2.10 (i), (ii) that

$$
\begin{aligned}
& d^{2}\left(x_{n+1}, \tilde{x}\right) \leq d^{2}\left(y_{n}, \tilde{x}\right)+2\left\langle\overrightarrow{x_{n+1} y_{n}}, \overrightarrow{x_{n+1}} \overrightarrow{\tilde{x}}\right\rangle \\
& \leq\left(\alpha_{n} d(\tilde{x}, \tilde{x})+\left(1-\alpha_{n}\right) d\left(T x_{n}, \tilde{x}\right)\right)^{2} \\
& +2\left[\alpha_{n}\left\langle\overrightarrow{f\left(x_{n}\right) y_{n}}, \overrightarrow{x_{n+1}} \overrightarrow{\tilde{x}}\right\rangle+\left(1-\alpha_{n}\right)\left\langle\overrightarrow{T x_{n} y_{n}}, \overrightarrow{x_{n+1}} \overrightarrow{\tilde{x}}\right\rangle\right] \\
& \leq\left(1-\alpha_{n}\right)^{2} d^{2}\left(x_{n}, \tilde{x}\right)+2\left[\alpha_{n} \alpha_{n}\left|\overrightarrow{f\left(x_{n}\right) \tilde{x}}, \overrightarrow{x_{n+1}} \overrightarrow{\tilde{x}}\right\rangle+\alpha_{n}\left(1-\alpha_{n}\right)\left|\overrightarrow{f\left(x_{n}\right) T x_{n}}, \overrightarrow{x_{n+1}} \overrightarrow{\tilde{x}}\right\rangle\right. \\
& \left.+\left(1-\alpha_{n}\right) \alpha_{n}\left\langle\overrightarrow{T x_{n} \tilde{x}}, \overrightarrow{x_{n+1}} \overrightarrow{\tilde{x}}\right\rangle+\left(1-\alpha_{n}\right)\left(1-\alpha_{n}\right)\left\langle\overrightarrow{T x_{n} T x_{n}}, \overrightarrow{x_{n+1}} \overrightarrow{\tilde{x}}\right\rangle\right] \\
& \leq\left(1-\alpha_{n}\right)^{2} d^{2}\left(x_{n}, \tilde{x}\right)+2\left[\alpha_{n} \alpha_{n}\left|\overrightarrow{f\left(x_{n}\right) \tilde{x}}, \overrightarrow{x_{n+1}} \overrightarrow{\tilde{x}}\right\rangle+\alpha_{n}\left(1-\alpha_{n}\right)\left|\overrightarrow{f\left(x_{n}\right) T x_{n}}, \overrightarrow{x_{n+1}} \overrightarrow{\tilde{x}}\right\rangle\right. \\
& \left.+\left(1-\alpha_{n}\right) \alpha_{n}\left\langle\overrightarrow{T x_{n} \tilde{x},} \overrightarrow{x_{n+1}} \vec{x}\right\rangle+\left(1-\alpha_{n}\right)^{2} d\left(T x_{n}, T x_{n}\right) d\left(x_{n+1} \tilde{x}\right)\right] \\
& =\left(1-\alpha_{n}\right)^{2} d^{2}\left(x_{n}, \tilde{x}\right)+2\left[\alpha_{n}^{2}\left|\overrightarrow{f\left(x_{n}\right)} \overrightarrow{\tilde{x}}, \overrightarrow{x_{n+1}} \overrightarrow{\tilde{x}}\right\rangle+\alpha_{n}\left(1-\alpha_{n}\right)\left\langle\overrightarrow{f\left(x_{n}\right) \tilde{x}}, \overrightarrow{x_{n+1}} \overrightarrow{\tilde{x}}\right\rangle\right] \\
& =\left(1-\alpha_{n}\right)^{2} d^{2}\left(x_{n}, \tilde{x}\right)+2 \alpha_{n}\left|\overrightarrow{f\left(x_{n}\right) \tilde{x}}, \overrightarrow{x_{n+1} \tilde{x}}\right\rangle \\
& =\left(1-\alpha_{n}\right)^{2} d^{2}\left(x_{n}, \tilde{x}\right)+2 \alpha_{n}\left|\overrightarrow{f\left(x_{n}\right) f(\tilde{x})}, \overrightarrow{x_{n+1} \tilde{x}}\right\rangle+2 \alpha_{n}\left|\overrightarrow{f(\tilde{x}) \tilde{x}}, \overrightarrow{x_{n+1}} \vec{x}\right\rangle \\
& \leq\left(1-\alpha_{n}\right)^{2} d^{2}\left(x_{n}, \tilde{x}\right)+2 \alpha_{n} \alpha d\left(x_{n}, \tilde{x}\right) d\left(x_{n+1}, \tilde{x}\right)+2 \alpha_{n}\left(\overrightarrow{f(\tilde{x})} \overrightarrow{\tilde{x}}, \overrightarrow{x_{n+1}} \overrightarrow{\tilde{x}}\right) \\
& \leq\left(1-\alpha_{n}\right)^{2} d^{2}\left(x_{n}, \tilde{x}\right)+\alpha_{n} \alpha\left(d^{2}\left(x_{n}, \tilde{x}\right)+d^{2}\left(x_{n+1}, \tilde{x}\right)\right)+2 \alpha_{n}\left\langle\overrightarrow{f(\tilde{x}) \tilde{x}}, \overrightarrow{x_{n+1}} \overrightarrow{\tilde{x}}\right\rangle,
\end{aligned}
$$

which implies that

$$
\begin{aligned}
d^{2}\left(x_{n+1}, \tilde{x}\right) & \leq \frac{1-(2-\alpha) \alpha_{n}+\alpha_{n}^{2}}{1-\alpha \alpha_{n}} d^{2}\left(x_{n}, \tilde{x}\right)+\frac{2 \alpha_{n}}{1-\alpha \alpha_{n}}\left\langle\overrightarrow{f(\tilde{x}) \tilde{x}}, \overrightarrow{x_{n+1} \tilde{x}}\right\rangle \\
& \leq \frac{1-(2-\alpha) \alpha_{n}}{1-\alpha \alpha_{n}} d^{2}\left(x_{n}, \tilde{x}\right)+\frac{2 \alpha_{n}}{1-\alpha \alpha_{n}}\left|\overrightarrow{f(\tilde{x}) \tilde{x}}, \overrightarrow{x_{n+1} \tilde{x}}\right\rangle+\alpha_{n}^{2} M,
\end{aligned}
$$

where $M \geq \sup _{n \geq 0}\left\{d^{2}\left(x_{n}, \tilde{x}\right)\right\}$. It then follows that

$$
d^{2}\left(x_{n+1}, \tilde{x}\right) \leq\left(1-\alpha_{n}^{\prime}\right) d^{2}\left(x_{n}, \tilde{x}\right)+\alpha_{n}^{\prime} \beta_{n}^{\prime},
$$


where

$$
\alpha_{n}^{\prime}=\frac{2(1-\alpha) \alpha_{n}}{1-\alpha \alpha_{n}} \quad \text { and } \quad \beta_{n}^{\prime}=\frac{\left(1-\alpha \alpha_{n}\right) \alpha_{n}}{2(1-\alpha)} M+\frac{1}{(1-\alpha)}\left\langle\overrightarrow{f(\vec{x})} \vec{x}, \overrightarrow{x_{n+1}} \overrightarrow{\tilde{x}}\right|
$$

Applying Lemma 2.8, we can conclude that $x_{n} \rightarrow \tilde{x}$. This completes the proof.

Remark 3.5 We give the different proof of [16, Theorem 2.3]. In fact, the property $\mathcal{P}$ imposed on a CAT(0) space $X$ is removed.

If $f \equiv u$, then the following corollary can be obtained directly from Theorem 3.4.

Corollary 3.6 [15, Theorem 2.3] Let $C$ be a closed convex subset of a complete CAT(0) space $X$, and let $T: C \rightarrow C$ be a nonexpansive mapping with $F(T) \neq \emptyset$. Let $u, x_{0} \in C$ be arbitrarily chosen and $\left\{x_{n}\right\}$ be generated by

$$
x_{n+1}=\alpha_{n} u \oplus\left(1-\alpha_{n}\right) T x_{n}, \quad \forall n \geq 0,
$$

where $\left\{\alpha_{n}\right\} \subset(0,1)$ satisfies the following conditions:

(i) $\lim _{n \rightarrow \infty} \alpha_{n}=0$;

(ii) $\sum_{n=0}^{\infty} \alpha_{n}=\infty$;

(iii) either $\sum_{n=0}^{\infty}\left|\alpha_{n+1}-\alpha_{n}\right|<\infty$ or $\lim _{n \rightarrow \infty}\left(\alpha_{n+1} / \alpha_{n}\right)=1$.

Then $\left\{x_{n}\right\}$ converges strongly as $n \rightarrow \infty$ to $\tilde{x} \in F(T)$ which is nearest to $u$ which is equivalent to the following variational inequality (18).

\section{Competing interests}

The authors declare that they have no competing interests.

\section{Authors' contributions}

Both authors read and approved the final manuscript.

\section{Acknowledgements}

The first author is supported by Naresuan university.

Received: 28 November 2012 Accepted: 9 February 2013 Published: 6 March 2013

\section{References}

1. Bruhat, F, Tits, J: Groupes ré ductifs sur un corps local. I. Donêes radicielles valuées. Inst. Hautes Êtudes Sci. Publ. Math. 41, 5-251 (1972)

2. Bridson, M, Haefliger, A: Metric Spaces of Nonpositive Curvature. Springer, Berlin (1999)

3. Brown, KS: Buildings. Springer, New York (1989)

4. Goebel, K, Reich, S: Uniform Convexity, Hyperbolic Geometry, and Nonexpansive Mappings. Series of Monographs and Textbooks in Pure and Applied Mathematics, vol. 83. Dekker, New York (1984)

5. Berg, ID, Nikolaev, IG: Quasilinearization and curvature of Alexandrov spaces. Geom. Dedic. 133, 195-218 (2008)

6. Kakavandi, BA, Amini, M: Duality and subdifferential for convex functions on complete CAT(0) metric spaces. Nonlinear Anal. 73, 3450-3455 (2010)

7. Dehghan, H, Rooin, J: A characterization of metric projection in CAT(0) spaces. In: International Conference on Functional Equation, Geometric Functions and Applications (ICFGA 2012), 10-12th May 2012, Payame Noor University, Tabriz, Iran, pp. 41-43 (2012)

8. Kirk, WA: Geodesic geometry and fixed point theory. In: Seminar of Mathematical Analysis (Malaga/Seville, 2002/2003), pp. 195-225. Colecc. Abierta, vol. 64. University of Seville, Secretary Publication, Seville (2003)

9. Banach, S: Sur les oṕration dans les ensembles abstraits et leur applications aux équations intégrales. Fundam. Math. 3, 133-181 (1922)

10. Cho, YJ, Ciric, LB, Wang, S: Convergence theorems for nonexpansive semigroups in CAT(0) spaces. Nonlinear Anal. 74 6050-6059 (2011)

11. Dhompongsa, S, Panyanak, B: On $\Delta$-convergence theorems in CAT(0) spaces. Comput. Math. Appl. 56, 2572-2579 (2008) 
12. Dhompongsa, S, Kaewkhao, A, Panyanak, B: Lim's theorems for multivalued mappings in CAT(0) spaces. J. Math. Anal. Appl. 312, 478-487 (2005)

13. Dhompongsa, S, Kirk, WA, Panyanak, B: Nonexpansive set-valued mappings in metric and Banach spaces. J. Nonlinear Convex Anal. 8, 35-45 (2007)

14. Dhompongsa, S, Kirk, WA, Sims, B: Fixed points of uniformly Lipschitzian mappings. Nonlinear Anal. 65, 762-772 (2006)

15. Saejung, S: Halpern's iteration in CAT(0) spaces. Fixed Point Theory Appl. 2010, 471781 (2010) doi:10.1155/2010/471781

16. Shi, LY, Chen, RD: Strong convergence of viscosity approximation methods for nonexpansive mappings in CAT(0) spaces. J. Appl. Math. 2012, 421050 (2012). doi:10.1155/2012/421050

17. Lim, TC: Remarks on some fixed point theorems. Proc. Am. Math. Soc. 60, 179-182 (1976)

18. Kirk, WA, Panyanak, B: A concept of convergence in geodesic spaces. Nonlinear Anal. 68, 3689-3696 (2008)

19. Kakavandi, BA: Weak topologies in complete CAT(0) metric spaces. Proc. Am. Math. Soc. 141(3), 1029-1039 (2013). doi:10.1090/S0002-9939-2012-11743-5

20. Xu, HK: An iterative approach to quadratic optimization. J. Optim. Theory Appl. 116, 659-678 (2003)

doi:10.1186/1029-242X-2013-93

Cite this article as: Wangkeeree and Preechasilp: Viscosity approximation methods for nonexpansive mappings in CAT(0) spaces. Journal of Inequalities and Applications 2013 2013:93.

\section{Submit your manuscript to a SpringerOpen ${ }^{\ominus}$ journal and benefit from:}

- Convenient online submission

Rigorous peer review

- Immediate publication on acceptance

Open access: articles freely available online

- High visibility within the field

- Retaining the copyright to your article 